\title{
Epistaxis issue search strategies
}

\section{Epistaxis and patient factors}

All databases searched 12th February 2016, no limits applied.

\section{Medline via Ovid \\ 1. exp Epistaxis/ \\ 2. (epistax* or nosebleed* or rhinorrhag* or rhinorrhaeg*).ti,ab. \\ 3. $\exp$ Nose/ \\ 4. (nose* or nasal ${ }^{*}$ or oronasal or nostril* ${ }^{*}$ or nasopharynx* or olfactory).ti,ab. \\ 5. 3 or 4 \\ 6. exp Hemorrhage/ \\ 7. (hemorrhag* or haemorrhag* or bleed* or bloodloss* or (blood adj3 loss)).ti,ab.}

8. 6 or 7

9. 5 and 8

10. 1 or 2 or 9

11. ((Discharg* ${ }^{*}$ or readmi* or (bed adj2 (day or days)) or inpatient* or outpatient* or ambulatory or recurren* or re-haemorrhag* or rehemorrhag* or rehaemorrhag* or rehemorrhag* or recurr* or operate* or operation* or comorbid* or multi-morbid* or ((pre-surg* or pre-operat* or presurg* or preoperat*) adj3 (assess* or care or procedure*)) or morbidity or complication* or transfus* or mortality or death or surgery or surgical or operation* or theatre* or operative or persist* or repeat or rebleed or (length adj1 stay) or LOS or admission or (number adj3 days) or overnight*).ti,ab.

12. exp inpatient/

13. exp patient discharge/

14. exp patient admission/

15. exp patient readmission/

16. exp length of stay/

17. exp outpatients/

18. exp outpatient clinics, hospital/

19. exp ambulatory care/

20. exp recurrence/

21. exp general surgery/

22. exp surgical procedures, operative/

23. exp postoperative complications/

24. exp preoperative care/

25. exp mortality/
26. exp morbidity/

27. exp comorbidity/

28. exp hospitalization/

29. or $/ 11-28$

30. (hospital ${ }^{*}$ or outcome* or risk* or predict*).ti,ab.

31. exp treatment outcome/

32. exp risk factors/

33. exp forecasting/

34. or $/ 30-33$

35. 10 and 29 and 34

\section{Embase strategy}

1. exp epistaxis/

2. (epistax* ${ }^{*}$ or nosebleed* ${ }^{*}$ or rhinorrhag* ${ }^{*}$ or rhinorrhaeg*).ti,ab.

3. exp nose/

4. (nose* $^{*}$ or nasal* or nostril* or nasopharyn* or olfactory).ti,ab.

5. 3 or 4

6. exp bleeding/

7. (hemorrhag* or haemorrhag* or bleed* or bloodloss* or (blood adj3 loss)).ti,ab.

8. 6 or 7

9. 5 and 8

10. 1 or 2 or 9

11. (Discharg* or readmi* or (bed adj2 (day or days)) or inpatient* or outpatient* or ambulatory or recurren* or re-haemorrhag* or rehemorrhag* or rehaemorrhag* or rehemorrhag* or recurr* or operate* or operation* or comorbid* $^{*}$ or multi-morbid* or ((pre-surg* or pre-operat* or presurg* or preoperat*) adj3 (assess* or care or procedure*)) or morbidity or complication* or transfus* or mortality or death or surgery or surgical or operation* or theatre* or operative or persist* or repeat or rebleed or (length adj1 stay) or LOS or admission or (number adj3 days) or overnight*).ti,ab.

12. 10 and 11

\section{Cochrane Database of Systematic Reviews}

1. MeSH descriptor: [Epistaxis] explode all trees

2. epistax* or nosebleed* or rhinorrhag* or rhinorrhaeg*:ti,ab,kw 
3. MeSH descriptor: [Nose] explode all trees

4. nose* or nasal* or oronasal or nostril* or nasopharynx* or olfactory:ti,ab,kw

5. \#3 or \#4

6. MeSH descriptor: [Hemorrhage] explode all trees

7. hemorrhag* or haemorrhag* or bleed* or bloodloss* or (blood near loss):ti,ab,kw

8. \#6 or \#7

9. \#5 and \#8

10. \#1 or \#2 or \#9

11. (Discharg* or readmi* or (bed near (day or days)) or inpatient* or outpatient* or ambulatory or recurren* or re-haemorrhag* or rehemorrhag* or rehaemorrhag* or rehemorrhag* or recurr* or operate* or operation* or comorbid $^{*}$ or multi-morbid* or ((pre-surg* or pre-operat* or presurg* or preoperat*) near (assess* ${ }^{*}$ or care or procedure*)) or morbidity or complication* or transfus* or mortality or death or surgery or surgical or operation* or theatre* $^{*}$ or operative or persist* or repeat or rebleed or (length near stay) or LOS or admission or (number near days) or overnight*)

12. \#10 and \#11

\section{CINAHL via EbscoHost}

1. (MH "Epistaxis")

2. TI (epistax* or nosebleed* ${ }^{*}$ or rhinorrhag* ${ }^{*}$ or rhinorrhaeg*) OR AB (epistax* or nosebleed* or rhinorrhag* or rhinorrhaeg*)

3. (MH "Nose+")

4. TI (nose* $^{*}$ or nasal* or oronasal or nostril ${ }^{*}$ or nasopharynx* or olfactory) $\mathrm{OR} \mathrm{AB}$ (nose* or nasal $^{*}$ or oronasal or nostril* or nasopharynx* or olfactory)

5. S3 OR S4

6. (MH "Hemorrhage+")

7. TI (hemorrhag* or haemorrhag* or bleed* or bloodloss* $^{*}$ or (blood N3 loss)) OR AB (hemorrhag* or haemorrhag* or bleed* or bloodloss* or (blood N3 loss))

8. S6 OR S7

9. S5 AND S8

10. S1 OR S2 OR S9

11. TI ((Discharg* or readmi* or (bed Near2 (day or days)) or inpatient* or outpatient* or ambulatory or recurren* or re-haemorrhag* or rehemorrhag* or rehaemorrhag* or rehemorrhag* or recurr* or operate* or operation* ${ }^{*}$ or comorbid* $^{*}$ or multi-morbid* ${ }^{*}$ or ((pre-surg* or pre-operat* or presurg* or preoperat*) Near3 (assess* or care or procedure*)) or morbidity or complication* ${ }^{*}$ or transfus* ${ }^{*}$ or mortality or death or surgery or surgical or operation* or theatre* or operative or persist* or repeat or rebleed or (length Near2 stay) or LOS or admission or (number Near2 days) or overnight*))
OR AB ((Discharg* or readmi* or (bed Near2 (day or days)) or inpatient* or outpatient* or ambulatory or recurren* or re-haemorrhag* or re-hemorrhag* or rehaemorrhag* or rehemorrhag* or recurr* or operate* or operation* $^{*}$ or co-morbid* or multi-morbid* or ((pre-surg* or pre-operat* or presurg* or pre-

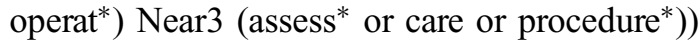
or morbidity or complication* or transfus* or mortality or death or surgery or surgical or operation* or theatre* or operative or persist* or repeat or re-bleed or (length Near2 stay) or LOS or admission or (number Near2 days) or overnight*))

12. (MH "Patient Discharge+")

13. $\mathrm{MH}$ "Readmission")

14. (MH "Bed Occupancy")

15. (MH "Inpatients")

16. (MH "Ambulatory Care")

17. (MH "Recurrence")

18. (MH "Surgery, Operative+")

19. (MH "Comorbidity")

20. (MH "Morbidity")

21. (MH "Mortality+")

22. (MH "Length of Stay")

23. (MH "Preoperative Care+")

24. (MH "Perioperative Care + "

25. (MH "Blood Transfusion+")

26. S11 OR S12 OR S13 OR S14 OR S15 OR S16 OR S17 OR S18 OR S19 OR S20 OR S21 OR S22 OR S23 OR S24 OR S25

27. S10 AND S26

Web of Science

1. $\mathrm{TS}=\left(\right.$ epistax $^{*}$ or nosebleed ${ }^{*}$ or rhinorrhag* or rhinorrhaeg* or ((nose* or nasal* or oronasal or nostril* or nasopharynx* or olfactory) and (hemorrhag* or haemorrhag* or bleed* or bloodloss* or (blood N/3 loss)) ))

2. $\mathrm{TS}=\left(\right.$ Discharg* $^{*}$ or readmi* or (bed N/2 (day or days)) or inpatient* or outpatient* or ambulatory or recurren* or re-haemorrhag* or rehemorrhag* or rehaemorrhag* or rehemorrhag* or recurr* or operate* or operation* or comorbid* $^{*}$ or multi-morbid* or ((pre-surg* or pre-operat* or presurg* or preoperat*) N/3 (assess ${ }^{*}$ or care or procedure*)) or morbidity or complication* or transfus* or mortality or death or surgery or surgical or operation* or theatre* or operative or persist* or repeat or rebleed or (length $\mathrm{N} / 2$ stay) or LOS or admission or (number $\mathrm{N} / 2$ days) or overnight*)

3. 1 and 2

\section{Epistaxis and initial assessment}

All databases searched 18th February 2016, no limits applied. 
Medline via Ovid

1. exp Epistaxis/

2. (epistax* or nosebleed* or rhinorrhag* or rhinorrhaeg*).ti,ab.

3. exp Nose/

4. (nose* $^{*}$ or nasal ${ }^{*}$ or oronasal or nostril ${ }^{*}$ or nasopharynx* or olfactory).ti,ab.

5. 3 or 4

6. exp Hemorrhage/

7. (hemorrhag* or haemorrhag* or bleed* or bloodloss* or (blood adj3 loss)).ti,ab.

8. 6 or 7

9. 5 and 8

10. 1 or 2 or 9

11. ((init* adj2 manag*) or history or present* or examin* or "universal precautions*" or "face mask" or (eye* adj2 protect*) or assess* or resuscitat* or observ* or "vital sign"” or triag* or investigat* or test* or diagnos* or endoscop* or thudicum or speculum or decongest* or cophenylcaine or xylometazoline or lignocaine or lidocaine or phenylephrine).ti,ab.

12. 10 and 11

\section{Embase strategy}

1. exp epistaxis/

2. (epistax* or nosebleed* or rhinorrhag* or rhinorrhaeg*).ti,ab.

3. exp nose/

4. (nose* $^{*}$ or nasal* or nostril* or nasopharyn* or olfactory).ti,ab.

5. 3 or 4

6. exp bleeding/

7. (hemorrhag* or haemorrhag* or bleed* or bloodloss* or (blood adj3 loss)).ti,ab.

8. 6 or 7

9. 5 and 8

10. 1 or 2 or 9

11. Xylometazoline (Embase Subject heading)

12. phenylephrine (Embase Subject heading)

13. lidocaine (Embase Subject heading)

14. ((init* adj2 manag*) or history or present* or examin* or "universal precautions" or "face mask" or (eye* adj2 protect*) or assess* or resuscitat* or observ* or "vital sign*" or triag* or investigat* or test* or diagnos* or endoscop* or thudicum or speculum or decongest* or cophenylcaine or xylometazoline or lignocaine or lidocaine or phenylephrine).ti,ab.

15. 10 and 11 and 12 and 13 and 14

\section{Cochrane Database of Systematic Reviews}

1. MeSH descriptor: [Epistaxis] explode all trees

2. epistax* or nosebleed* or rhinorrhag* or rhinorrhaeg*:ti,ab,kw

3. MeSH descriptor: [Nose] explode all trees
4. nose* ${ }^{*}$ or nasal* or oronasal or nostril* or nasopharynx* or olfactory:ti,ab,kw

5. \#3 or \#4

6. MeSH descriptor: [Hemorrhage] explode all trees

7. hemorrhag* or haemorrhag* or bleed* or bloodloss* or (blood near loss):ti,ab,kw

8. \#6 or \#7

9. \#5 and \#8

10. \#1 or \#2 or \#9

11. ("initial* manag*" or "manag* initial"" or examin* or "universal precautions" or "face mask" or (eye* adj2 protect*) or assess* or resuscitat* or observ* or "vital sign*" or triag* or investigat* or test* or "work up" or diagnos* or endoscop* or thudicum or speculum or decongest* or co-phenylcaine or xylometazoline or lignocaine or lidocaine or phenylephrine):ti,ab,kw

12. \#10 and \#11

\section{CINAHL via EbscoHost}

1. (MH "Epistaxis")

2. TI (epistax* or nosebleed* or rhinorrhag* or rhinorrhaeg*) OR AB (epistax* or nosebleed* or rhinorrhag* or rhinorrhaeg*)

3. (MH "Nose+")

4. TI (nose* or nasal* or oronasal or nostril* or nasopharynx* or olfactory) OR AB (nose* or nasal* $^{*}$ or oronasal or nostril* or nasopharynx* or olfactory)

5. S3 OR S4

6. (MH "Hemorrhage+")

7. TI (hemorrhag* or haemorrhag* or bleed* or bloodloss* or (blood N3 loss)) OR AB (hemorrhag* or haemorrhag* or bleed* or bloodloss* or (blood N3 loss))

8. S6 OR S7

9. S5 AND S8

10. S1 OR S2 OR S9

11. TI(initial ${ }^{*} \mathrm{~N} 3$ manag* $^{*}$ OR AB (initial $^{*} \mathrm{~N} 3$ manag*)

12. TI(history or present* or examin* or "universal precautions" " or "face mask" or (eye* N2 protect*) or assess* or resuscitat* or observ* or "vital sign*" or triag* or investigat* or test* or diagnos* or endoscop* or thudicum or speculum or decongest* or co-phenylcaine or xylometazoline or lignocaine or lidocaine or phenylephrine) OR AB (history or present* or examin* or "universal precautions*" or "face mask" or (eye* N2 protect*) or assess* or resuscitat* or observ* or "vital sign*" or triag* or investigat* or test* or diagnos* or endoscop* or thudicum or speculum or decongest* or co-phenylcaine or xylometazoline or lignocaine or lidocaine or phenylephrine)

13. S11 OR S12

14. S10 AND S13 


\section{Epistaxis and first aid}

All databases searched 11th February 2016, no limits applied.

\section{Medline via Ovid}

1. exp Epistaxis/

2. (epistax* or nosebleed ${ }^{*}$ or rhinorrhag* or rhinorrhaeg*).ti,ab.

3. $\exp$ Nose/

4. (nose* $^{*}$ or nasal* or oronasal or nostril ${ }^{*}$ or nasopharynx* or olfactory).ti,ab.

5. 3 or 4

6. exp Hemorrhage/

7. (hemorrhag* or haemorrhag* or bleed* or bloodloss* or (blood adj3 loss)).ti,ab.

8. 6 or 7

9. 5 and 8

10. 1 or 2 or 9

11. exp First Aid

12. ("first aid" or or ice or compress* or pressure or tamponade or irrigate* or suction* or pinch* or position* or posture or clip or ice or cold or cooling or airway or minors or majors or "resuscitation room" or nurs* or clot or lean*or hippocratic).ti,ab.

13. trotter.mp

14. 11 or 12 or 13

15. 10 and 14

\section{Embase strategy}

1. exp epistaxis/

2. (epistax* or nosebleed* ${ }^{*}$ or rhinorrhag* or rhinorrhaeg*).ti,ab.

3. $\exp$ nose/

4. (nose* or nasal* or nostril* or nasopharyn* or olfactory).ti,ab.

5. 3 or 4

6. exp bleeding/

7. (hemorrhag* or haemorrhag* or bleed* or bloodloss* or (blood adj3 loss)).ti,ab.

8. 6 or 7

9. 5 and 8

10. 1 or 2 or 9

11. ("first aid" or or ice or compress* or pressure or tamponade or irrigate* or suction* or pinch* or position* or posture or clip or ice or cold or cooling or airway or minors or majors or "resuscitation room" or nurs* or clot or lean*or hippocratic).ti,ab.

12. Trotter.mp

13. 10 and 11 and 12

\section{Cochrane Database of Systematic Reviews}

1. MeSH descriptor: [Epistaxis] explode all trees

2. epistax* $^{*}$ or nosebleed* or rhinorrhag* or rhinorrhaeg*:ti,ab,kw

3. MeSH descriptor: [Nose] explode all trees
4. nose* or nasal* or oronasal or nostril* or nasopharynx* or olfactory:ti,ab,kw

5. \#3 or \#4

6. MeSH descriptor: [Hemorrhage] explode all trees

7. hemorrhag* or haemorrhag* or bleed* or bloodloss* or (blood near loss):ti,ab,kw

8. \#6 or \#7

9. $\# 5$ and $\# 8$

10. $\# 1$ or $\# 2$ or $\# 9$

11. ("first aid" or ice or compress* or pressure or tamponade or irrigate* or suction* or pinch* ${ }^{*}$ or position* or posture or clip or ice or cold or cooling or airway or minors or majors or "resuscitation room" or nurs* or clot or lean* or trotter or Hippocratic):ti,ab,kw

12. \#10 and \#11

\section{CINAHL via EbscoHost}

1. (MH "Epistaxis")

2. TI (epistax* or nosebleed* or rhinorrhag* or rhinorrhaeg*) OR AB (epistax* or nosebleed* or rhinorrhag* or rhinorrhaeg*)

3. (MH "Nose+")

4. TI (nose* $^{*}$ or nasal* or oronasal or nostril* or nasopharynx* or olfactory) OR AB (nose* or nasal $^{*}$ or oronasal or nostril* or nasopharynx* or olfactory)

5. S3 OR S4

6. (MH "Hemorrhage+")

7. TI (hemorrhag* or haemorrhag* or bleed* or bloodloss* or (blood N3 loss)) OR AB (hemorrhag* or haemorrhag* or bleed* or bloodloss* or (blood N3 loss))

8. S6 OR S7

9. S5 AND S8

10. S1 OR S2 OR S9

11. (MH "First Aid+")

12. TI("first aid" or ice or compress* or pressure or tamponade or irrigate* or suction* or pinch* or position* or posture or clip or ice or cold or cooling or airway or minors or majors or "resuscitation room" or clot or lean* or trotter or Hippocratic) OR AB ("first aid" or ice or compress* or pressure or tamponade or irrigate* or suction* or pinch* or position* or posture or clip or ice or cold or cooling or airway or minors or majors or "resuscitation room" or clot or lean* or trotter or Hippocratic)

13. S11 OR S12

14. S10 AND S13

\section{Epistaxis and cautery}

All databases searched 12th February 2016, no limits applied.

\section{Medline via Ovid}

1. exp Epistaxis/ 
2. (epistax* or nosebleed* or rhinorrhag* or rhinorrhaeg*).ti,ab.

3. exp Nose/

4. nose* $^{*}$ or nasal ${ }^{*}$ or oronasal or nostril ${ }^{*}$ or nasopharynx* or olfactory).ti,ab.

5. 3 or 4

6. exp Hemorrhage/

7. (hemorrhag* or haemorrhag* or bleed* or bloodloss* or (blood adj3 loss)).ti,ab.

8. 6 or 7

9. 5 and 8

10. 1 or 2 or 9

11. exp Cautery or (cauter* or electrocauter* or "electr* cauter*" or electrocoagulat* or "electr* coagulat" " or electrosurg* or "electr* surg*" or "silver nitrate" or "chemical cauter" or bipolar or diatherm* or "septal perforation" or (septum adj3 perforat") or AgNO3 or "suction monopolar" or "hot wir").ti,ab.

12. 10 and 11

\section{Embase strategy}

1. exp epistaxis/

2. (epistax* or nosebleed* or rhinorrhag* or rhinorrhaeg*).ti,ab.

3. exp nose/

4. (nose* or nasal $^{*}$ or nostril ${ }^{*}$ or nasopharyn* or olfactory).ti,ab.

5. 3 or 4

6. exp bleeding/

7. (hemorrhag* or haemorrhag* or bleed* or bloodloss* or (blood adj3 loss)).ti,ab.

8. 6 or 7

9. 5 and 8

10. 1 or 2 or 9

11. Exp cauterisation or (cauter* or electrocauter* or "electr* cauter*" or electrocoagulat* or "electr* coagulat" " or electrosurg* or "electr* surg*" or "silver nitrate" or "chemical cauter" or bipolar or diatherm* or "septal perforation" or (septum adj3 perforat") or AgNO3 or "suction monopolar" or "hot wir"”).ti,ab

12. 10 and 11

\section{Cochrane Database of Systematic Reviews}

1. MeSH descriptor: [Epistaxis] explode all trees

2. epistax* or nosebleed* or rhinorrhag* or rhinorrhaeg*:ti,ab,kw

3. MeSH descriptor: [Nose] explode all trees

4. nose* or nasal ${ }^{*}$ or oronasal or nostril ${ }^{*}$ or nasopharynx* or olfactory:ti,ab,kw

5. \#3 or \#4

6. MeSH descriptor: [Hemorrhage] explode all trees

7. hemorrhag* or haemorrhag* or bleed* or bloodloss* or (blood near loss):ti,ab,kw

8. \#6 or \#7

9. \#5 and \#8
10. $\# 1$ or $\# 2$ or $\# 9$

11. (cauter* or electrocauter* or "electr* cauter*" or electrocoagulat* or "electr* coagulat" or electrosurgery or "electr* surgery" or "silver nitrate" or bipolar or diatherm* or suction* or monopolar or "hot wir"" or AgNO3):ti,ab,kw

12. \#10 and \#11

\section{CINAHL via EbscoHost}

1. (MH "Epistaxis")

2. TI (epistax* or nosebleed* or rhinorrhag* or rhinorrhaeg*) OR AB (epistax* or nosebleed* or rhinorrhag* or rhinorrhaeg*)

3. (MH "Nose+")

4. TI (nose* or nasal ${ }^{*}$ or oronasal or nostril ${ }^{*}$ or nasopharyn $x^{*}$ or olfactory) OR AB (nose* or nasal* $^{*}$ or oronasal or nostril* ${ }^{*}$ or nasopharynx* or olfactory)

5. S3 OR S4

6. (MH "Hemorrhage+")

7. TI (hemorrhag* or haemorrhag* or bleed* or bloodloss* or (blood N3 loss)) OR AB (hemorrhag* or haemorrhag* or bleed* or bloodloss* or (blood N3 loss))

8. S6 OR S7

9. S5 AND S8

10. S1 OR S2 OR S9

11. TI(cauter* or electrocauter* or "electr* cauter*" or electrocoagulat* or "electr* coagulat" or electrosurgery or "electr* surgery" or "silver nitrate" or bipolar or diatherm* or suction* or monopolar or "hot wir"" or AgNO3)OR AB (cauter* or electrocauter* or "electr* cauter*" or electrocoagulat* or "electr* coagulat" or electrosurgery or "electr* surgery" or "silver nitrate" or bipolar or diatherm* or suction* or monopolar or "hot wir*" or AgNO3)

12. S10 AND S11

\section{Epistaxis and non-dissolvable packing}

All databases searched 12th February 2016, no limits applied.

\section{Medline via Ovid}

1. exp Epistaxis/

2. (epistax* or nosebleed* or rhinorrhag* or rhinorrhaeg*).ti,ab.

3. exp Nose/

4. (nose* $^{*}$ or nasal ${ }^{*}$ or oronasal or nostril ${ }^{*}$ or nasopharynx* or olfactory).ti,ab.

5. 3 or 4

6. exp Hemorrhage/

7. (hemorrhag* or haemorrhag* or bleed* or bloodloss* or (blood adj3 loss)).ti,ab.

8. 6 or 7

9. 5 and 8

10. 1 or 2 or 9 
11. (non-biodegrad* or nonbiodegrad* or nondissovl* $^{*}$ or non-dissolv* or remov* or inflat* or tampon* or balloon* or Foley or catheter* or ribbon* or gauze* or tribromophenate or bismuth* or iodoform* or iodine* or paraffin* or BIPP or foam or PVA or PVAc or (polyvinyl adj alcohol) or (polyvinyl adj acetal) or Merocel or Expandacell or ultracell or ultraplus or (Rapid adj rhino) or Doyle or AbsorbENT or EpiStax or Netcell or Pak or (Rhino adj rocket) or epi-stop or epi-max or Xeroform or Raucocel or blu or (Series adj 5000) or Netcell or (liquifilm adj tears)).ti,ab.

12. exp tampons, surgical/

13. exp balloon occlusion/

14. exp catheters/

15. exp polyvinyl alcohol/

16. exp surgical sponges/

17. exp bismuth/

18. exp Endotamponade/

19. or $/ 11-18$

20. 10 and 19

\section{Embase strategy}

1. exp epistaxis/

2. (epistax* or nosebleed* ${ }^{*}$ or rhinorrhag* or rhinorrhaeg*).ti,ab.

3. exp nose/

4. (nose* or nasal* or nostril* or nasopharyn* ${ }^{*}$ or olfactory).ti,ab.

5. 3 or 4

6. exp bleeding/

7. (hemorrhag* or haemorrhag* or bleed* or bloodloss* or (blood adj3 loss)).ti,ab.

8. 6 or 7

9. 5 and 8

10. 1 or 2 or 9

11. (non-biodegrad* or nonbiodegrad* or nondissovl $^{*}$ or non-dissolv* or remov* or inflat* or tampon* or balloon* or Foley or catheter* or ribbon* or gauze* or tribromophenate or bismuth* or iodoform* or iodine* or paraffin* or BIPP or foam or PVA or PVAc or (polyvinyl adj alcohol) or (polyvinyl adj acetal) or Merocel or Expandacell or ultracell or ultraplus or (Rapid adj rhino) or Doyle or AbsorbENT or EpiStax or Netcell or Pak or (Rhino adj rocket) or epi-stop or epi-max or Xeroform or Raucocel or blu or (Series adj 5000) or Netcell or (liquifilm adj tears)).ti,ab.

12. 10 and 11

\section{Cochrane Database of Systematic Reviews}

1. MeSH descriptor: [Epistaxis] explode all trees

2. epistax* or nosebleed* or rhinorrhag* or $^{*}$ rhinorrhaeg*:ti,ab,kw

3. MeSH descriptor: [Nose] explode all trees
4. nose* or nasal* or oronasal or nostril* or nasopharynx* or olfactory:ti,ab,kw

5. \#3 or \#4

6. MeSH descriptor: [Hemorrhage] explode all trees

7. hemorrhag* or haemorrhag* or bleed* or bloodloss* or (blood near loss):ti,ab,kw

8. \#6 or \#7

9. $\# 5$ and $\# 8$

10. $\# 1$ or $\# 2$ or $\# 9$

11. (non-biodegrad* or nonbiodegrad* or nondissovl $^{*}$ or non-dissolv* ${ }^{*}$ or remov* or inflat* or tampon* or balloon* or Foley or catheter* or ribbon* or gauze* or tribromophenate or bismuth* $^{*}$ or iodoform* or iodine* or paraffin* or BIPP or foam or PVA or PVAc or (polyvinyl near alcohol) or (polyvinyl near acetal) or Merocel or Expandacell or ultracell or ultraplus or (Rapid near rhino) or Doyle or AbsorbENT or EpiStax or Netcell or Pak or (Rhino near rocket) or epi-stop or epi-max or Xeroform or Raucocel or blu or (Series near 5000) or Netcell or (liquifilm near tears)):ti,ab,kw

12. \#10 and \#11

\section{CINAHL via EbscoHost}

1. (MH "Epistaxis")

2. TI (epistax* or nosebleed* or rhinorrhag* or rhinorrhaeg*) OR AB (epistax* or nosebleed* or rhinorrhag* or rhinorrhaeg*)

3. (MH "Nose+")

4. TI (nose* $^{*}$ or nasal* or oronasal or nostril ${ }^{*}$ or nasopharynx* or olfactory) OR AB (nose* or nasal $^{*}$ or oronasal or nostril* or nasopharynx* or olfactory)

5. S3 OR S4

6. (MH "Hemorrhage+")

7. TI (hemorrhag* or haemorrhag* or bleed* or bloodloss* or (blood N3 loss)) OR AB (hemorrhag* or haemorrhag* or bleed* or bloodloss* or (blood N3 loss))

8. S6 OR S7

9. S5 AND S8

10. $\mathrm{S} 1$ OR S2 OR S9

11. TI ((non-biodegrad* or nonbiodegrad* or nondissovl $^{*}$ or non-dissolv* or remov* or inflat* or tampon* or balloon* or Foley or catheter* or ribbon* or gauze* or tribromophenate or bismuth* or iodoform* or iodine* or paraffin* or BIPP or foam or PVA or PVAc or (polyvinyl near alcohol) or (polyvinyl near acetal) or Merocel or Expandacell or ultracell or ultraplus or (Rapid near rhino) or Doyle or AbsorbENT or EpiStax or Netcell or Pak or (Rhino near rocket) or epi-stop or epi-max or Xeroform or Raucocel or blu or (Series near 5000) or Netcell or (liquifilm near tears)):) OR AB ((non-biodegrad* or nonbiodegrad* or 
nondissovl* or non-dissolv* or remov* or inflat* or tampon* or balloon* or Foley or catheter* or ribbon* or gauze* or tribromophenate or bismuth* or iodoform* or iodine* or paraffin* or BIPP or foam or PVA or PVAc or (polyvinyl near alcohol) or (polyvinyl near acetal) or Merocel or Expandacell or ultracell or ultraplus or (Rapid near rhino) or Doyle or AbsorbENT or EpiStax or Netcell or Pak or (Rhino near rocket) or epi-stop or epi-max or Xeroform or Raucocel or blu or (Series near 5000) or Netcell or (liquifilm near tears))

12. (MH "Balloon Dilatation+")

13. (MH "Nasal Packing")

14. (MH "Gauze Dressings")

15. (MH "Bismuth")

16. (MH "Paraffin")

17. (MH "polyvinals")

18. (MH "tampons")

19. S11 OR S12 OR S13 OR S14 OR S15 OR S16 OR S17 OR S18

20. S10 AND S19

Web of Science

1. $\mathrm{TS}=$ epistax $^{*}$ or nosebleed ${ }^{*}$ or rhinorrhag* ${ }^{*}$ or rhinorrhaeg* or ((nose* or nasal* or oronasal or nostril* or nasopharynx* or olfactory) and (hemorrhag* or haemorrhag* or bleed* or bloodloss* or (blood N/3 loss))))

2. $\mathrm{TS}=(($ non-biodegrad* or nonbiodegrad* or nondissovl* or non-dissolv* or remov* or inflat* or tampon* or balloon* or Foley or catheter* or ribbon* or gauze* or tribromophenate or bismuth* or iodoform* or iodine* or paraffin* or BIPP or foam or PVA or PVAc or (polyvinyl $\mathrm{N} / 1$ alcohol) or (polyvinyl N/1 acetal) or Merocel or Expandacell or ultracell or ultraplus or (Rapid N/1 rhino) or Doyle or AbsorbENT or EpiStax or Netcell or Pak or (Rhino N/1 rocket) or epi-stop or epi-max or Xeroform or Raucocel or blu or (Series N/1 5000) or Netcell or (liquifilm adj tears)))

3. 1 and 2

\section{Epistaxis and dissolvable packing}

All databases searched 11th February 2016, no limits applied.

\section{Medline via Ovid}

1. exp Epistaxis/

2. (epistax* or nosebleed* or rhinorrhag* or rhinorrhaeg*).ti,ab.

3. $\exp$ Nose/

4. (nose $^{*}$ or nasal ${ }^{*}$ or oronasal or nostril* ${ }^{*}$ or nasopharynx* or olfactory).ti,ab.

5. 3 or 4

6. exp Hemorrhage/
7. (hemorrhag* or haemorrhag* or bleed* or bloodloss* or (blood adj3 loss)).ti,ab.

8. 6 or 7

9. 5 and 8

10. 1 or 2 or 9

11. (absor* OR dissolv* OR bioabsor* OR bioabsor* OR bioresor* OR bio-resor* OR resorb* OR resorp* OR biodegrad* OR "biodegrad*" OR biofragment* OR "bio-fragment*" OR fragment* OR "non-removable" OR "haemostatic agent*" OR "hemostatic agent*" OR (haemostatic* adj 2 seal $^{*}$ ) OR (hemostatic* adj3 seal*) OR "haemostatic agent*" OR "hemostatic agent*" OR "haemostatic matri*" OR "hemostatic matri*" OR nasopore OR "polyurethane foam" OR meropack OR merogel OR hyaluron* OR cutanplast OR "Stammberger Sinus Dressin"” OR "sinu-foam" OR "sinu foam" OR "sinu-knit" OR Carboxymethylcellulose OR Quixil OR (Fibrin* adj3 (glue* or adhesive* or seal $^{*}$ )) OR crosseal OR transglue* OR quickclot OR "Microporous polysaccharide hemosphere*" OR "mucopolysaccharide hemosphere*" OR "Microporous polysaccharide hemisphere*" OR mucopolysaccharide OR Chitosan* OR floseal OR surgiflo OR "gelatin* matri*" OR hemostase OR "posi-sep" OR posisep OR kaltostat OR

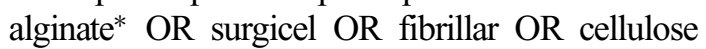
OR epicell OR chitohem OR evarrest OR "fibrin sealant" OR traumastem).mp.

12. exp Absorbable Implants/

13. exp cellulose/

14. exp chitosan/

15. exp hemostasis/

16. exp hemostatics/

17. exp fibrin foam/

18. exp gelatin sponge, absorbable/

19. exp Fibrin Tissue Adhesive/

20. exp Epirubicin/

21. exp Carboxymethylcellulose Sodium/

22. exp Biocompatible Materials /

23. exp Polyurethanes/

24. exp Alginates/

25. exp Glycosaminoglycans /

26. or $/ 11-25$

27. 10 and 26

\section{Embase strategy}

1. exp epistaxis/

2. (epistax* or nosebleed* or rhinorrhag* ${ }^{*}$ or rhinorrhaeg*).ti,ab.

3. exp nose/

4. nose* $^{*}$ or nasal* or nostril ${ }^{*}$ or nasopharyn* or olfactory).ti,ab.

5. 3 or 4

6. exp bleeding/

7. (hemorrhag* or haemorrhag* or bleed* or bloodloss* or (blood adj3 loss)).ti,ab. 
8. 6 or 7

9. 5 and 8

10. 1 or 2 or 9

11. (absor* OR dissolv* OR bioabsor* OR bioabsor* OR bioresor* OR bio-resor* OR resorb* OR resorp* OR biodegrad* OR "biodegrad*" OR biofragment* OR "bio-fragment*" OR fragment* OR "non-removable" OR "haemostatic agent*" OR "hemostatic agent" OR (haemostatic* adj 2 seal*) OR (hemostatic* adj3 seal*) OR "haemostatic agent"" OR "hemostatic agent"" OR "haemostatic matri*" OR "hemostatic matri*" OR nasopore OR "polyurethane foam" OR meropack OR merogel OR hyaluron* OR cutanplast OR "Stammberger Sinus Dressin*" OR "sinufoam" OR "sinu foam" OR "sinu-knit" OR Carboxymethylcellulose OR Quixil OR (Fibrin* adj3 (glue* or adhesive* or seal*)) OR crosseal OR transglue* OR quickclot OR "Microporous polysaccharide hemosphere*" OR "mucopolysaccharide hemosphere*" OR "Microporous polysaccharide hemisphere*" OR mucopolysaccharide OR Chitosan* OR floseal OR surgiflo OR "gelatin* matri*" OR hemostase OR “posi-sep” OR posisep OR kaltostat OR alginate* OR surgicel OR fibrillar OR cellulose OR epicell OR chitohem OR evarrest OR "fibrin sealant" OR traumastem).ti,ab.

12. 10 and 11

Cochrane Database of Systematic Reviews

1. MeSH descriptor: [Epistaxis] explode all trees

2. epistax* or nosebleed* or rhinorrhag* or rhinorrhaeg*:ti,ab,kw

3. MeSH descriptor: [Nose] explode all trees

4. nose* or nasal* or oronasal or nostril* or nasopharynx* or olfactory:ti,ab,kw

5. \#3 or \#4

6. MeSH descriptor: [Hemorrhage] explode all trees

7. hemorrhag* or haemorrhag* or bleed* or bloodloss* or (blood near loss):ti,ab,kw

8. \#6 or \#7

9. $\# 5$ and $\# 8$

10. $\# 1$ or $\# 2$ or \#9

11. (absor* OR dissolv* OR bioabsor* OR bioabsor* OR bioresor* OR bio-resor* OR resorb* OR resorp* OR biodegrad* OR "biodegrad*" OR biofragment* OR "bio-fragment*" OR fragment* OR "non-removable" OR "haemostatic agent*" OR "hemostatic agent"" OR (haemostatic* NEAR 2 seal*) OR (hemostatic* NEAR seal*) OR "haemostatic agent"" OR "hemostatic agent*" OR "haemostatic matri*" OR "hemostatic matri*" OR nasopore OR "polyurethane foam" OR meropack OR merogel OR hyaluron* OR cutanplast OR
"Stammberger Sinus Dressin*" OR "sinufoam" OR "sinu foam" OR "sinu-knit" OR Carboxymethylcellulose OR Quixil OR (Fibrin* NEAR (glue* or adhesive* or seal*)) OR crosseal OR transglue* OR quickclot OR "Microporous polysaccharide hemosphere*" OR "mucopolysaccharide hemosphere*" OR "Microporous polysaccharide hemisphere*" OR mucopolysaccharide OR Chitosan* OR floseal OR surgiflo OR "gelatin* matri*" OR hemostase OR “posi-sep" OR posisep OR kaltostat OR alginate* OR surgicel OR fibrillar OR cellulose OR epicell OR chitohem OR evarrest OR “fibrin sealant" OR traumastem)):ti,ab,kw (Word variations have been searched)

12. \#10 and \#12

\section{CINAHL via EbscoHost}

1. (MH "Epistaxis")

2. TI (epistax* or nosebleed* or rhinorrhag* or rhinorrhaeg*) OR AB (epistax* or nosebleed* or rhinorrhag* or rhinorrhaeg*)

3. (MH "Nose+")

4. TI (nose* or nasal* or oronasal or nostril* or nasopharynx* or olfactory) OR AB (nose* or nasal $^{*}$ or oronasal or nostril* or nasopharynx* or olfactory)

5. S3 OR S4

6. (MH "Hemorrhage+")

7. TI (hemorrhag* or haemorrhag* or bleed* or bloodloss* or (blood N3 loss)) OR AB (hemorrhag* $^{*}$ or haemorrhag* or bleed* ${ }^{*}$ or bloodloss* or (blood N3 loss))

8. S6 OR S7

9. S5 AND S8

10. S1 OR S2 OR S9

11. TI (absor* OR dissolv* OR bioabsor* OR bioabsor* OR bioresor* OR bio-resor* OR resorb* OR resorp* OR biodegrad* OR "biodegrad*" OR biofragment* OR "bio-fragment*" OR fragment* OR "non-removable" OR "haemostatic agent"" OR "hemostatic agent*" OR (haemostatic* NEAR 2 seal*) OR (hemostatic* NEAR seal*) OR "haemostatic agent*" OR "hemostatic agent*" OR "haemostatic matri*" OR "hemostatic matri*" OR nasopore OR "polyurethane foam" OR meropack OR merogel OR hyaluron* OR cutanplast OR "Stammberger Sinus Dressin*" OR "sinufoam" OR "sinu foam" OR "sinu-knit" OR Carboxymethylcellulose OR Quixil OR (Fibrin* NEAR (glue* or adhesive* or seal*)) OR crosseal OR transglue* OR quickclot OR "Microporous polysaccharide hemosphere*" OR "mucopolysaccharide hemosphere*" OR "Microporous polysaccharide hemisphere*" OR mucopolysaccharide OR Chitosan* OR floseal OR surgiflo OR "gelatin* matri*" OR 
hemostase OR "posi-sep" OR posisep OR kaltostat OR alginate* OR surgicel OR fibrillar OR cellulose OR epicell OR chitohem OR evarrest OR "fibrin sealant" OR traumastem) $O R$ AB (absor* OR dissolv* OR bioabsor* OR bioabsor* OR bioresor* OR bio-resor* OR resorb* OR resorp* OR biodegrad* OR "biodegrad*" OR biofragment* OR "bio-fragment"” OR fragment* OR "non-removable" $O R$ "haemostatic agent" OR "hemostatic agent" OR (haemostatic* NEAR 2 seal ${ }^{*}$ ) OR (hemostatic* NEAR seal*) OR "haemostatic agent"" OR "hemostatic agent" OR "haemostatic matri"” OR "hemostatic matri*" OR nasopore OR "polyurethane foam" OR meropack OR merogel OR hyaluron* OR cutanplast OR "Stammberger Sinus Dressin"” OR "sinufoam" OR "sinu foam" OR "sinu-knit" OR Carboxymethylcellulose OR Quixil OR (Fibrin* NEAR (glue* or adhesive* or seal*)) OR crosseal OR transglue* OR quickclot OR "Microporous polysaccharide hemosphere*" OR "mucopolysaccharide hemosphere*" OR "Microporous polysaccharide hemisphere*" OR mucopolysaccharide OR Chitosan* OR floseal OR surgiflo OR "gelatin* matri*" OR hemostase OR "posi-sep" OR posisep OR kaltostat OR alginate* OR surgicel OR fibrillar OR cellulose OR epicell OR chitohem OR evarrest OR "fibrin sealant" OR traumastem)

12. (MH "Cellulose")

13. (MH "Hemostasis+")

14. (MH "Hemostatics+")

15. (MH "Epirubicin")

16. (MH "Biocompatible Materials +")

17. (MH "Polyurethanes")

18. (MH "Glycosaminoglycans+")

19. (MH "Fibrin+")

20. (MH "Fibrin Tissue Adhesive")

21. S11 or S12 OR S13 OR S14 OR S15 OR S16 OR S17 OR S18 OR S19 OR S20

22. S10 AND S21

Web of Science

1. $T s=\left(e^{2} i s t a x^{*}\right.$ or nosebleed* or rhinorrhag* or rhinorrhaeg* or ((nose* or nasal* or oronasal or nostril* or nasopharynx* or olfactory) and (hemorrhag* or haemorrhag* or bleed* or bloodloss* or (blood N/3 loss))))

2. $\mathrm{TS}=$ (absor $^{*}$ OR dissolv* OR bioabsor* $\mathrm{OR}$ bio-absor* OR bioresor* OR bio-resor* OR resorb* OR resorp* OR biodegrad* OR "biodegrad $^{*}$ " OR biofragment" OR "bio-fragment*" OR fragment* OR "non-removable")

3. $\mathrm{TS}=\left(\right.$ hemostatic $^{*} \mathrm{NEAR} / 3$ seal $\left.^{*}\right)$ OR $\mathrm{TS}=$ (hemostatic* NEAR/3 agent*)

4. $\mathrm{TS}=$ (nasopore $\mathrm{OR}$ "polyurethane foam" OR meropack OR merogel OR hyaluron* OR cutanplast OR "Stammberger Sinus Dressin*" OR "sinu-foam" OR "sinu foam" OR "sinuknit" OR Carboxymethylcellulose OR Quixil)

5. $\mathrm{TS}=\left(\right.$ Fibrin $\left.^{*} \mathrm{NEAR} / 3 \mathrm{seal}^{*}\right)$

6. $\mathrm{TS}=($ crosseal OR transglue* $\mathrm{OR}$ quickclot $\mathrm{OR}$ "Microporous polysaccharide hemosphere" OR "mucopolysaccharide hemosphere" "Microporous polysaccharide hemisphere*" OR mucopolysaccharide OR Chitosan* OR floseal OR surgiflo OR "gelatin* matri"” OR hemostase OR "posi-sep" OR posisep OR kaltostat OR alginate* OR surgicel OR fibrillar OR cellulose OR epicell OR chitohem OR evarrest OR traumastem)

7. $\mathrm{TS}=\left(\right.$ fibrin near $/ 3$ glue $\left.{ }^{*}\right)$

8. $\mathrm{TS}=\left(\right.$ fibrin near $/ 3$ adhesiv $\left.{ }^{*}\right)$

9. $\mathrm{TS}=\left(\right.$ haemostatic ${ }^{*}$ near $/ 3$ matri)

10. $\mathrm{TS}=\left(\right.$ haemostatic $^{*}$ near $/ 3$ seal $\left.^{*}\right)$

11. $\mathrm{TS}=\left(\right.$ haemostatic* near $/ 3$ agent $\left.{ }^{*}\right)$

12. $\mathrm{TS}=\left(\right.$ hemostatic ${ }^{*} \mathrm{NEAR} / 3$ matri* $)$

13. 2 or 3 or 4 or 5 or 6 or 7 or 8 or 9 or 10 or 11 or 12

14. 1 and 13

\section{Epistaxis and warfarin}

All databases searched 11th February 2016, no limits applied.

\section{Medline via Ovid}

1. exp Epistaxis/

2. (epistax* or nosebleed* or rhinorrhag* or rhinorrhaeg*).ti,ab.

3. $\exp$ Nose/

4. (nose* or nasal ${ }^{*}$ or oronasal or nostril* or nasopharynx* or olfactory).ti,ab.

5. 3 or 4

6. exp Hemorrhage/

7. (hemorrhag* or haemorrhag* or bleed* or bloodloss* or (blood adj3 loss)).ti,ab.

8. 6 or 7

9. 5 and 8

10. 1 or 2 or 9

11. exp Warfarin

12. exp coumarins

13. exp vitamin $\mathrm{K}$

14. (inhibitor* or antagonist*).ti,ab

15. 13 and 14

16. exp International normalized ratio

17. (high or reversal).ti,ab.

18. 16 and 17

19. (coumarin or "vitamin $\mathrm{k}$ inhibitor" " or "vitamin k antagonist*" or "high inr" or "reversal inr" or Coumadin or "internal normali" ratio").ti,ab.

20. 11 or 12 or 15 or 18 or 19

21. 10 and 20

Embase strategy

1. exp epistaxis/ 
2. (epistax* or nosebleed* or rhinorrhag* or rhinorrhaeg*).ti,ab.

3. $\exp$ nose/

4. (nose* or nasal* or nostril* or nasopharyn* or olfactory).ti,ab.

5. 3 or 4

6. exp bleeding/

7. (hemorrhag* or haemorrhag* or bleed* or bloodloss* or (blood adj3 loss)).ti,ab.

8. 6 or 7

9. 5 and 8

10. 1 or 2 or 9

11. ("vitamin $\mathrm{k}$ inhibitor*" or "vitamin $\mathrm{k}$ antagonist*" or "high inr" or "reversal inr" or Coumadin or "international normali* ratio").ti,ab.

12. Warfarin (Embase Subject heading)

13. Coumarin (Embase Subject heading)

14. Antivitamin k (Embase Subject heading)

15. 11 or 12 or 13 or 14

16. 10 and 15

\section{Cochrane Database of Systematic Reviews}

1. MeSH descriptor: [Epistaxis] explode all trees

2. epistax* or nosebleed* or rhinorrhag* or rhinorrhaeg*:ti,ab,kw

3. MeSH descriptor: [Nose] explode all trees

4. nose* or nasal* or oronasal or nostril* or nasopharynx* or olfactory:ti,ab,kw

5. \#3 or \#4

6. MeSH descriptor: [Hemorrhage] explode all trees

7. hemorrhag* or haemorrhag* or bleed* or bloodloss* or (blood near loss):ti,ab,kw

8. \#6 or \#7

9. $\# 5$ and $\# 8$

10. \#1 or \#2 or \#9

11. (warfarin or coumarin or "vitamin $\mathrm{k}$ inhibitor*" or "vitamin k antagonist"" or "high inr" or "reversal inr" or Coumadin or "internal normali* ratio"):ti,ab,kw

12. \#10 and \#11

\section{CINAHL via EbscoHost}

1. (MH "Epistaxis")

2. TI (epistax* or nosebleed* or rhinorrhag* or rhinorrhaeg*) OR AB (epistax* or nosebleed* or rhinorrhag* or rhinorrhaeg*)

3. (MH "Nose+")

4. TI (nose* or nasal* or oronasal or nostril* or nasopharynx* or olfactory) OR AB (nose* or nasal* $^{*}$ or oronasal or nostril* ${ }^{*}$ or nasopharynx* or olfactory)

5. S3 OR S4

6. (MH "Hemorrhage+")

7. TI (hemorrhag* or haemorrhag* or bleed* or bloodloss* or (blood N3 loss)) OR $\mathrm{AB}$ (hemorrhag* or haemorrhag* or bleed* or bloodloss* or (blood N3 loss))
8. S6 OR S7
9. S5 AND S8

10. S1 OR S2 OR S9

11. (MH "Warfarin")

12. (MH"Vitamin k")

13. TI(inhibitor* or antagonist*)OR AB (inhibitor* or antagonist*)

14. S12 AND S13

15. MH("International normalised ratio")

16. TI(high or reversal) OR $\mathrm{AB}$ ((high or reversal)

17. S15 AND S16

18. TI(coumarin or "vitamin $\mathrm{k}$ inhibitor" or "vitamin k antagonist*" or "high inr" or "reversal inr" or Coumadin or "internal normali" ratio") $\mathrm{OR} \mathrm{AB}$ (coumarin or "vitamin k inhibitor"" or "vitamin k antagonist"" or "high inr" or "reversal inr" or Coumadin or "internal normali* ratio")

19. S11 OR S14 OR S18

20. S10 AND S19

\section{Epistaxis and novel oral anticoagulants}

All databases searched 12th February 2016, no limits applied.

\section{Medline via Ovid}

1. exp Epistaxis/

2. (epistax* or nosebleed* or rhinorrhag* or rhinorrhaeg*).ti,ab.

3. $\exp$ Nose/

4. (nose* or nasal ${ }^{*}$ or oronasal or nostril* ${ }^{*}$ or nasopharynx* or olfactory).ti,ab.

5. 3 or 4

6. exp Hemorrhage/

7. (hemorrhag* or haemorrhag* or bleed* or bloodloss* or (blood adj3 loss)).ti,ab.

8. 6 or 7

9. 5 and 8

10. 1 or 2 or 9

11. Exp rivarozaban or exp dabigatran or exp factor xa inhibitors or xarelto or pradaxa or apixaban or eliquis or ximelagatran or exanta or exoxaban or lixiana or (factor adj3 inhibitor*) or (factor adj3 antagonist*) or fondaparinux or "thrombin inhibitor"" or "thrombin antagonist*" or "novel anticoagulant" or "new anticoagulant*" or "novel oral anticoagulant" or noac or ldarucizumab

12. 10 and 11

Embase strategy

1. exp epistaxis/

2. (epistax* or nosebleed* or rhinorrhag* ${ }^{*}$ or rhinorrhaeg*).ti,ab.

3. exp nose/ 
4. (nose* or nasal* or nostril* or nasopharyn* or olfactory).ti,ab.

5. 3 or 4

6. exp bleeding/

7. (hemorrhag* or haemorrhag* or bleed* or bloodloss* or (blood adj3 loss)).ti,ab.

8. 6 or 7

9. 5 and 8

10. 1 or 2 or 9

11. Exp rivaroxaban or exp dabigatran or exp factor xa inhibitors or xarelto or pradaxa or apixaban or eliquis or ximelagatran or exanta or exoxaban or lixiana or (factor adj3 inhibitor*) or (factor adj3 antagonist*) or fondaparinux or "thrombin inhibitor*" or "thrombin antagonist*" or "novel anticoagulant*" or "new anticoagulant*" or "novel oral anticoagulant*" or noac or ldarucizumab

12. 10 and 11

Cochrane Database of Systematic Reviews

1. MeSH descriptor: [Epistaxis] explode all trees

2. epistax* $^{*}$ or nosebleed* or rhinorrhag* or rhinorrhaeg*:ti,ab,kw

3. MeSH descriptor: [Nose] explode all trees

4. nose* or nasal* or oronasal or nostril* or nasopharynx* or olfactory:ti,ab,kw

5. \#3 or \#4

6. MeSH descriptor: [Hemorrhage] explode all trees

7. hemorrhag* or haemorrhag* or bleed* or bloodloss* or (blood near loss):ti,ab,kw

8. \#6 or \#7

9. \#5 and \#8

10. \#1 or \#2 or \#9

11. (rivarozaban or dabigatran or xarelto or pradaxa or apixaban or eliquis or ximelagatran or exanta or exoxaban or lixiana or (factor adj3 inhibitor*) or (factor adj3 antagonist*) or fondaparinux or "thrombin inhibitor" " or "thrombin antagonist*" or "novel anticoagulant" or "new anticoagulant*" or "novel oral anticoagulant*" or noac or ldarucizumab):ti,ab,kw

12. 10 and 11

\section{CINAHL via EbscoHost}

1. (MH "Epistaxis")

2. TI (epistax* or nosebleed* or rhinorrhag* or rhinorrhaeg*) OR AB (epistax* or nosebleed* or rhinorrhag* or rhinorrhaeg*)

3. (MH "Nose+")

4. TI (nose* or nasal* or oronasal or nostril ${ }^{*}$ or nasopharynx* or olfactory) OR AB (nose* or nasal* $^{*}$ or oronasal or nostril* or nasopharynx* or olfactory)

5. S3 OR S4

6. (MH "Hemorrhage+")
7. TI (hemorrhag* or haemorrhag* or bleed* or bloodloss* or (blood N3 loss)) OR AB (hemorrhag* or haemorrhag* $^{*}$ or bleed* or bloodloss* or (blood N3 loss))

8. S6 OR S7

9. S5 AND S8

10. S1 OR S2 OR S9

11. TI(rivarozaban or dabigatran or xarelto or pradaxa or apixaban or eliquis or ximelagatran or exanta or exoxaban or lixiana or (factor adj3 inhibitor*) or (factor adj3 antagonist*) or fondaparinux or "thrombin inhibitor" ${ }^{*}$ or "thrombin antagonist*" or "novel anticoagulant*" or "new anticoagulant*" or "novel oral anticoagulant*" or noac or ldarucizumab) OR AB (rivarozaban or dabigatran or xarelto or pradaxa or apixaban or eliquis or ximelagatran or exanta or exoxaban or lixiana or (factor adj3 inhibitor*) or (factor adj3 antagonist*) or fondaparinux or "thrombin inhibitor*" or "thrombin antagonist"" or "novel anticoagulant*" or "new anticoagulant*" or "novel oral anticoagulant*" or noac or ldarucizumab

12. S10 AND S11

\section{Epistaxis and heparin}

All databases searched 12th February 2016, no limits applied.

\section{Medline via Ovid}

1. exp Epistaxis/

2. (epistax* or nosebleed* or rhinorrhag* or rhinorrhaeg*).ti,ab.

3. $\exp$ Nose/

4. nose* $^{*}$ or nasal ${ }^{*}$ or oronasal or nostril ${ }^{*}$ or nasopharynx* or olfactory).ti,ab.

5. 3 or 4

6. exp Hemorrhage/

7. (hemorrhag* or haemorrhag* or bleed* or bloodloss* or (blood adj3 loss)).ti,ab.

8. 6 or 7

9. 5 and 8

10. 1 or 2 or 9

11. exp dalteparin or exp protamines or or "low molecular weight heprin" or APTT* or "activated partial thromboplastin time" or antithromb* or ATIII or glycosaminoglycan or thrombocyto* or HITTs or "heparin induced thrombosis" or clexane or enoxaparin or tinzaparin or dalteparin or protamine or lepirudin or desirudin or bivalirudin or argatroban

12. 10 and 11

\section{Embase strategy}

1. exp epistaxis/

2. (epistax* or nosebleed* ${ }^{*}$ or rhinorrhag* or rhinorrhaeg*).ti,ab.

3. exp nose/ 
4. (nose* or nasal* or nostril* or nasopharyn* or olfactory).ti,ab.

5. 3 or 4

6. $\exp$ bleeding/

7. (hemorrhag* or haemorrhag* or bleed* or bloodloss* or (blood adj3 loss)).ti,ab.

8. 6 or 7

9. 5 and 8

10. 1 or 2 or 9

11. exp enoxaparin or exp lepirudin or heparin(MESH) or exp tinzaparin or exp desulfatohirudin or exp hirulog or exp argatroban or exp dalteparin or exp protamine or (heparin* or heprin or APTT* or "activated partial thromboplastin time" or antithrom* or ATIII or glycosaminoglycan or thromb* or HITTs or clexane or desirudin or bivalirudin).

12. 10 and 11

\section{Cochrane Database of Systematic Reviews}

1. MeSH descriptor: [Epistaxis] explode all trees

2. epistax $^{*}$ or nosebleed* or rhinorrhag* or rhinorrhaeg*:ti,ab,kw

3. MeSH descriptor: [Nose] explode all trees

4. nose* or nasal* or oronasal or nostril* or nasopharynx* or olfactory:ti,ab,kw

5. \#3 or \#4

6. MeSH descriptor: [Hemorrhage] explode all trees

7. hemorrhag* or haemorrhag* or bleed* or bloodloss* or (blood near loss):ti,ab,kw

8. \#6 or \#7

9. \#5 and \#8

10. \#1 or \#2 or \#9

11. (heparin* or "low molecular weight heprin" or APTT* or "activated partial thromboplastin time" or antithromb* or ATIII or glycosaminoglycan or thrombocyto* or HITTs or "heparin induced thrombosis" or clexane or enoxaparin or tinzaparin or dalteparin or protamine or lepirudin or desirudin or bivalirudin or argatroban):ti,ab,kw

12. \#10 and \#11

\section{CINAHL via EbscoHost}

1. (MH "Epistaxis")

2. TI (epistax* or nosebleed* ${ }^{*}$ or rhinorrhag* or rhinorrhaeg*) OR AB (epistax* or nosebleed* or rhinorrhag* or rhinorrhaeg*)

3. (MH "Nose+")

4. TI (nose* $^{*}$ or nasal* or oronasal or nostril ${ }^{*}$ or nasopharynx* or olfactory) OR AB (nose* or nasal $^{*}$ or oronasal or nostril* or nasopharynx ${ }^{*}$ or olfactory)

5. S3 OR S4

6. (MH "Hemorrhage+")

7. TI (hemorrhag* or haemorrhag* or bleed* or bloodloss* or (blood N3 loss)) OR AB (hemorrhag* or haemorrhag* or bleed* or bloodloss* or (blood N3 loss))

8. S6 OR S7

9. S5 AND S8

10. S1 OR S2 OR S9

11. (MH heparin+ or bivalirudin+ or argatroban+ or lepirudin + )

12. TI(heparin* or heprin or aptt* or "activated partial thromboplastin time" or antithromb* or ATIII or glycosaminoglycan or thromb* or hitts or clexane or tinzaparin or desirudin) OR AB (heparin* or heprin or aptt* or "activated partial thromboplastin time" or antithromb* or ATIII or glycosaminoglycan or thromb* or hitts or clexane or tinzaparin or desirudin)

13. S11 OR S12

14. S10 AND 13

\section{Epistaxis and antiplatelet therapy}

All databases searched 11th February 2016, no limits applied.

\section{Medline via Ovid}

1. exp Epistaxis/

2. (epistax* or nosebleed* ${ }^{*}$ or rhinorrhag* or rhinorrhaeg*).ti,ab.

3. $\exp$ Nose/

4. (nose* or nasal* or oronasal or nostril* or nasopharynx* or olfactory).ti,ab.

5. 3 or 4

6. exp Hemorrhage/

7. (hemorrhag* or haemorrhag* or bleed* or bloodloss* or (blood adj3 loss)).ti,ab.

8. 6 or 7

9. 5 and 8

10. 1 or 2 or 9

11. exp Platelet aggregation inhibitors

12. (antiplatelet* or clopidogrel or tirofiban).ti,ab.

13. exp Aspirin

14. exp Adenosine diphosphate

15. (inhibitor* or antagonist*).ti,ab.

16. 14 and 15

17. ((adp adj2 inhibitor*) or "adp antagonist*" or "thromboxane inhibitor*" or "platelet inhibitor*" or (adenosine adj2 inhibitor*) or "adenosine diphosphate antagonist*").ti,ab.

18. exp Dipyridamole

19. exp Ticlopidine

20. (eptifibatide or aggrastat or (blood adj2 thin*).ti,ab.

21. exp Cyclooxygenase inhibitors

22. ("cox inhibitor"" or "cox antagonist*" or gllbllla or "cyclooxigenase inhibitor"” or "cyclo-oxygenase antagonist*" or NSAID*).ti,ab.

23. exp Thienopyridines

24. exp Phosphoric diester hydrolases 
25. ("phosphodiesterase inhibitor*" or prasugrel or Plavix or brillinta or effient or ticagrelor or ticlid).ti,ab.

26. exp Prasugrel hydrochloride

27. exp Anti-inflammatory agents, non steroidal

28. (cilostazol or pletal or persantine or abciximab or reopro).ti,ab.

29. ((glycoprotein adj3 inhibitor*" or "glycoprotein llbllla antagonist*" or "pde inhibitor*" or "phosphodiesterase antagonist*" or "phosphodiesterase inhibitor"" or "pde inhibitor" or pde antagonist"').ti,ab.

30. 11 or 12 or 13 or 16 or 17 or 18 or 19 or 20 or 21 or 22 or 23 or 24 or 25 or 26 or 27 or 28 or 29

31. 10 and 30

\section{Embase strategy}

1. exp epistaxis/

2. (epistax* or nosebleed* or rhinorrhag* or rhinorrhaeg*).ti,ab.

3. exp nose/

4. (nose* $^{*}$ or nasal* or nostril* ${ }^{*}$ or nasopharyn* or olfactory).ti,ab.

5. 3 or 4

6. $\exp$ bleeding/

7. (hemorrhag* or haemorrhag* or bleed* or bloodloss* or (blood adj3 loss)).ti,ab.

8. 6 or 7

9. 5 and 8

10. 1 or 2 or 9

11. (antiplatelet* or "platelet inhibitor"" or "cyclooxygenase inhibitor*" or "cyclo-oxygenase antagonist" or "cox inhibitor*" or "cox antagonist*" or nsaid* or aspirin or "adp inhibitor" or "adp antagonist*" or "adenosine diphosphate inhibitor*" or "adenosine diphosphate antagonist*" or clopidogrel or Plavix or effient or brillinta or ticlopidine or ticlid or "phosphodiesterase inhibitor*" or "pde inhibitor*" or "phosphodiesterase antagonist*" or "pde antagonist*" or pletal or "adenosine reuptake inhibitor*" or persantine or thienopyridines or "glycoprotein 1lb/1lla inhibitor"" or "glycoprotein llbllla antagonist*" or "gllbllla inhibitor*" or "gllbllla antagonist*" or reopro or aggrastat).ti,ab.

12. non steroidal anti-inflammatory agent (Embase subject heading)

13. Clopidogrel (Embase subject heading)

14. Prasugrel (Embase subject heading)

15. Ticagrelor(Embase subject heading)

16. Antithrombocyclic agent(Embase subject heading)

17. Ticlopidine(Embase subject heading)

18. Cilostazol(Embase subject heading)

19. Dipyridamole(Embase subject heading)

20. Abciximab(Embase subject heading)
21. Eptifibatide(Embase subject heading)

22. 11 or 12 or 13 or 14 or 15 or 16 or 17 or 18 or 19 or 20 or 21

23. 10 and 22

Cochrane Database of Systematic Reviews

1. MeSH descriptor: [Epistaxis] explode all trees

2. epistax* $^{*}$ or nosebleed* or rhinorrhag* or rhinorrhaeg*:ti,ab,kw

3. MeSH descriptor: [Nose] explode all trees

4. nose* or nasal* or oronasal or nostril* or nasopharynx* or olfactory:ti,ab,kw

5. \#3 or \#4

6. MeSH descriptor: [Hemorrhage] explode all trees

7. hemorrhag* or haemorrhag* or bleed* or bloodloss* or (blood near loss):ti,ab,kw

8. \#6 or \#7

9. $\# 5$ and $\# 8$

10. $\# 1$ or $\# 2$ or $\# 9$

11. (antiplatelet* or "platelet inhibitor" or "cyclooxygenase inhibitor" or "cyclo-oxygenase antagonist*" or "cox inhibitor*" or "cox antagonist*" or "non steroidal anti-inflammator*" or nsaid* or aspirin or "adp inhibitor" or "adp antagonist*" or "adenosine diphosphate inhibitor*" or "adenosine diphosphate antagonist*" or clopidogrel or Plavix or prasugrel or effient or ticagrelor or brillinta or ticlopidine or ticlid or "phosphodiesterase inhibitor"" or "pde inhibitor*" or "phosphodiesterase antagonist*" or "pde antagonist*" or cilostazol or pletal or "adenosine reuptake inhibitor"" or dipyridamole or persantine or thienopyridines or "glycoprotein 1lb/1lla inhibitor"" or "glycoprotein llbllla antagonist*" or "gllbllla inhibitor*" or "gllbllla antagonist*" or abciximab or reopro or eptifibatide or aggrastat):ti,ab,kw

\section{CINAHL via EbscoHost}

1. (MH "Epistaxis")

2. TI (epistax* or nosebleed* or rhinorrhag* or rhinorrhaeg*) OR AB (epistax* or nosebleed* or rhinorrhag* or rhinorrhaeg*)

3. (MH "Nose+")

4. TI (nose* or nasal* or oronasal or nostril ${ }^{*}$ or nasopharynx* or olfactory) OR AB (nose* or nasal $^{*}$ or oronasal or nostril* or nasopharynx* or olfactory)

5. S3 OR S4

6. (MH "Hemorrhage+")

7. TI (hemorrhag* or haemorrhag* or bleed* or bloodloss* or (blood N3 loss)) OR AB (hemorrhag* or haemorrhag* or bleed* or bloodloss* or (blood N3 loss))

8. S6 OR S7 
9. S5 AND S8

10. S1 OR S2 OR S9

11. (MH "Platelet aggregation inhibitors")

12. (MH "Clopidogrel”)

13. (MH "Tirofiban")

14. TI(antiplatelet*)OR AB (antiplatelet*)

15. (MH "Aspirin")

16. (MH "Adenosine diphosphate")

17. TI(inhibitor* or antagonist*)OR AB (inhibitor* or antagonist*)

18. S16 AND S17

19. TI ((adp N2 inhibitor*) or "adp antagonist"” or "thromboxane inhibitor*" or "platelet inhibitor*" or (adenosine adj2 inhibitor*) or "adenosine diphosphate antagonist"”)OR AB ((adp N2 inhibitor*) or "adp antagonist*" or "thromboxane inhibitor*" or "platelet inhibitor*" or (adenosine adj2 inhibitor*) or "adenosine diphosphate antagonist*")

20. (MH "Dipyridamole")

21. (MH "Ticlopidine")

22. (MH "Eptifibatide")

23. TI (aggrastat) OR AB(aggrastat)

24. TI (blood N2 thin*)OR AB (blood N2 thin*)

25. TI ("cox inhibitor*" or "cox antagonist*" or gllbllla or "cyclooxigenase inhibitor*" or "cyclo-oxygenase antagonist"" or NSAID*)OR AB ("cox inhibitor"" or "cox antagonist"” or gllbllla or "cyclooxigenase inhibitor"" or "cyclo-oxygenase antagonist*" or NSAID*)

26. (MH "Phosphodiesterase inhibitors")

27. TI (Thienopyridine*)OR AB (Thienopyridine*)

28. (MH "Prasugrel Hydorchloride")

29. (MH "Abciximab")

30. TI (Prasugrel or Plavix or brillinta or effient or pletal or persantine or reopro)OR AB (Prasugrel or Plavix or brillinta or effient or pletal or persantine or reopro)

31. TI(ticagrelor or ticlid)OR AB (ticagrelor or ticlid)

32. (MH "Antiinflammatory agents, non-steroidal")

33. TI (Glycoprotein N3 inhibitor* or "glycoprotein llbllla antagonist*" or "pde inhibitor*" or "phosphodiesterase antagonist"" or "pde antagonist*" or "phosphodiesterase inhibitor") OR AB (Glycoprotein N3 inhibitor* or "glycoprotein llbllla antagonist*" or "pde inhibitor" or "phosphodiesterase antagonist*" or "pde antagonist*" or "phosphodiesterase inhibitor")

34. $\mathrm{S} 11$ or $\mathrm{S} 12$ or $\mathrm{S} 13$ or $\mathrm{S} 14$ or $\mathrm{S} 15$ or $\mathrm{S} 16$ or $\mathrm{S} 17$ or $\mathrm{S} 18$ or $\mathrm{S} 19$ or $\mathrm{S} 20$ or $\mathrm{S} 21$ or $\mathrm{S} 22$ or $\mathrm{S} 23$ or $\mathrm{S} 24$ or $\mathrm{S} 25$ or $\mathrm{S} 26$ or $\mathrm{S} 27$ or $\mathrm{S} 28$ or $\mathrm{S} 29$ or $\mathrm{S} 30$ or $\mathrm{S} 31$ or $\mathrm{S} 32$ or $\mathrm{S} 33$

35. S10 AND S34

\section{Epistaxis and transfused blood products}

All databases searched 12th February 2016, no limits applied.
Medline via Ovid

1. exp Epistaxis/

2. (epistax* or nosebleed* or rhinorrhag* or rhinorrhaeg*).ti,ab.

3. $\exp$ Nose/

4. (nose $^{*}$ or nasal* or oronasal or nostril* ${ }^{*}$ or nasopharynx* or olfactory).ti,ab.

5. 3 or 4 6 exp Hemorrhage/

7. (hemorrhag* or haemorrhag* or bleed* or bloodloss* or (blood adj3 loss)).ti,ab.

8. 6 or 7

9. 5 and 8

10. 1 or 2 or 9

11. (transfuse* or blood)

12. blood transfusion (MESH)

13. (clotting or "promthrombin concentrate" or platelets).ti,ab

14. (ffp or "fresh frozen plasma").ti,ab.

15. (cell* adj3 salvage*).ti,ab.

16. ("autologous donation" or "cross match" or "o negative" or "type specific").ti,ab.

17. (cryoprecipitate or clotting).ti,ab.

18. ("packed red cells" or "factor 7" or "factor seven" or "factor vii" or "factor VII").ti,ab.

19. ("low haemoglobin" or "low haemoglobin").ti,ab.

20. 11 or 12 or 13 or 14 or 15 or 16 or 17 or 18 or 19

21. 10 and 20

Embase strategy

1. exp epistaxis/

2. (epistax* or nosebleed* or rhinorrhag* or rhinorrhaeg*).ti,ab.

3. $\exp$ nose/

4. (nose* $^{*}$ or nasal* ${ }^{*}$ or nostril ${ }^{*}$ or nasopharyn* or olfactory).ti,ab.

5. 3 or 4

6. exp bleeding/

7. (hemorrhag* or haemorrhag* or bleed* or bloodloss* or (blood adj3 loss)).ti,ab.

8. 6 or 7

9. 5 and 8

10. 1 or 2 or 9

11. (clotting or "prothrombin concentrate" or platelets or ffp or "fresh frozen plasma" or haemoglobin or haemoglobin or (cell* adj3 salvage*) or "autologous donation" or "cross match" or "o negative" or "type specific" OR cryoprecipitate or fibrinogen or "packed red cells" or "factor 7 " or "factor seven" or "factor vii" or "factor VII").ti,ab. Or haemoglobin(Embase Subject heading)

12. 10 and 11

\section{Cochrane Database of Systematic Reviews}

1. MeSH descriptor: [Epistaxis] explode all trees

2. epistax* or nosebleed* or rhinorrhag* or rhinorrhaeg*:ti,ab,kw 
3. MeSH descriptor: [Nose] explode all trees

4. nose* or nasal* or oronasal or nostril* ${ }^{*}$ or nasopharynx* or olfactory:ti,ab,kw

5. \#3 or \#4

6. MeSH descriptor: [Hemorrhage] explode all trees

7. hemorrhag* or haemorrhag* or bleed* or bloodloss* or (blood near loss):ti,ab,kw

8. \#6 or \#7

9. $\# 5$ and $\# 8$

10. $\# 1$ or $\# 2$ or \#9

11. (transfus* or "packed red cells" or clotting or "prothrombin concentrate" or platelets or ffp or "fresh frozen plasma" or cryoprecipitate or "low haemoglobin" or "low haemoglobin" or "cell salvage" or "autologous donation" or "cross match" or "o negative" or "type specific" or "factor seven" or "factor 7" or "factor vii"):ti,ab,kw

12. \#10 and \#11

\section{CINAHL via EbscoHost}

1. (MH "Epistaxis")

2. TI (epistax* or nosebleed* or rhinorrhag* or rhinorrhaeg*) OR AB (epistax* or nosebleed* or rhinorrhag* or rhinorrhaeg*)

3. (MH "Nose+")

4. TI (nose* or nasal* or oronasal or nostril* or nasopharynx* or olfactory) OR AB (nose* or nasal* $^{*}$ or oronasal or nostril* or nasopharynx* or olfactory)

5. S3 OR S4

6. (MH "Hemorrhage+")

7. TI (hemorrhag* or haemorrhag* or bleed* or bloodloss* or (blood N3 loss)) OR AB (hemorrhag* or haemorrhag* or bleed* or bloodloss* or (blood N3 loss))

8. S6 OR S7

9. S5 AND S8

10. S1 OR S2 OR S9

11. TI(transfus* or "packed red cells" or clotting or "prothrombin concentrate" or platelets or ffp or "fresh frozen plasma" or cryoprecipitate or "low haemoglobin" or "low haemoglobin" or "cell salvage" or "autologous donation" or "cross match" or "o negative" or "type specific" or "factor seven" or "factor 7" or "factor vii")OR AB (transfus* or "packed red cells" or clotting or "prothrombin concentrate" or platelets or ffp or "fresh frozen plasma" or cryoprecipitate or "low haemoglobin" or "low haemoglobin" or "cell salvage" or "autologous donation" or "cross match" or "o negative" or "type specific" or "factor seven" or "factor 7" or "factor vii")

12. S10 and S11

\section{Epistaxis and tranexamic acid}

All databases searched 12th February 2016, no limits applied.

\section{Medline via Ovid}

1. exp Epistaxis/

2. (epistax* or nosebleed* ${ }^{*}$ or rhinorrhag* or rhinorrhaeg*).ti,ab.

3. $\exp$ Nose/

4. (nose* $^{*}$ or nasal ${ }^{*}$ or oronasal or nostril ${ }^{*}$ or nasopharynx* or olfactory).ti,ab.

5. 3 or 4

6. exp Hemorrhage/

7. (hemorrhag* or haemorrhag* or bleed* or bloodloss* or (blood adj3 loss)).ti,ab.

8. 6 or 7

9. 5 and 8

10. 1 or 2 or 9

11. Exp tranexamic acid or exp antifibrinolytic agents or txa or tranexamic or antifebrin* or (clot* adj3 stabili*) or cyklokapron

12. 10 and 11

Embase strategy

1. exp epistaxis/

2. (epistax* or nosebleed* or rhinorrhag* or rhinorrhaeg*).ti,ab.

3. exp nose/

4. (nose* $^{*}$ or nasal ${ }^{*}$ or nostril ${ }^{*}$ or nasopharyn* or olfactory).ti,ab.

5. 3 or 4

6. exp bleeding/

7. (hemorrhag* or haemorrhag* or bleed* or bloodloss* or (blood adj3 loss)).ti,ab.

8. 6 or 7

9. 5 and 8

10. 1 or 2 or 9

11. Exp tranexamic acid or exp antifibrinolytic agents or txa or tranexamic or antifebrin* or (clot* adj3 stabili*) or cyklokapron

12. 10 and 11

\section{Cochrane Database of Systematic Reviews}

1. MeSH descriptor: [Epistaxis] explode all trees

2. epistax* or nosebleed* or rhinorrhag* or rhinorrhaeg*:ti,ab,kw

3. MeSH descriptor: [Nose] explode all trees

4. nose* or nasal* or oronasal or nostril* or nasopharynx* or olfactory:ti,ab,kw

5. \#3 or \#4

6. MeSH descriptor: [Hemorrhage] explode all trees

7. hemorrhag* or haemorrhag* or bleed* or bloodloss* or (blood near loss):ti,ab,kw

8. \#6 or \#7

9. \#5 and \#8

10. \#1 or \#2 or \#9 
11. (txa or tranexamic or antifebrin* or "clot stabili*" or cyklokapron):ti,ab,kw

12. 10 and 11

\section{CINAHL via EbscoHost}

1. (MH "Epistaxis")

2. TI (epistax* or nosebleed* or rhinorrhag* or rhinorrhaeg*) OR AB (epistax* or nosebleed* or rhinorrhag* or rhinorrhaeg*)

3. $(\mathrm{MH}$ "Nose + ")

4. TI (nose* $^{*}$ or nasal* or oronasal or nostril ${ }^{*}$ or nasopharynx* or olfactory) OR AB (nose* or nasal* $^{*}$ or oronasal or nostril* or nasopharynx* or olfactory)

5. S3 OR S4

6. (MH "Hemorrhage+")

7. TI (hemorrhag* or haemorrhag* or bleed* or bloodloss* or (blood N3 loss)) OR AB (hemorrhag* or haemorrhag* $^{*}$ or bleed* or bloodloss* or (blood N3 loss))

8. S6 OR S7

9. S5 AND S8

10. S1 OR S2 OR S9

11. txa or tranexamic or antifebrin* or (clot adj3 stabili*) or cyklokapron

12. 10 and 11

\section{Epistaxis and surgery}

All databases searched 11th February 2016, no limits applied.

\section{Medline via Ovid}

1. exp Epistaxis/

2. (epistax* or nosebleed* or rhinorrhag* or rhinorrhaeg*).ti,ab.

3. $\exp$ Nose/

4. (nose* $^{*}$ or nasal* or oronasal or nostril* ${ }^{*}$ or nasopharynx* or olfactory).ti,ab.

5. 3 or 4

6. exp Hemorrhage/

7. (hemorrhag* or haemorrhag* or bleed* or bloodloss* or (blood adj3 loss)).ti,ab.

8. 6 or 7

9. 5 and 8

10. 1 or 2 or 9

11. (surger* or surgic* or operat* or ligat* or transoral $^{*}$ or transnasal* or Caldwell-luc* or diatherm* ${ }^{*}$ or post-operat* or pre-operat* or postsurg* or pre-surg* or sphenopalatine or ethmoid or (internal adj maxillary adj arter*) or (external adj carotid adj arter*) or clip* or staple* or laser*).ti,ab.

12. exp general surgery/

13. exp surgical procedures, operative/

14. exp postoperative period/

15. exp preoperative period/

16. exp postoperative care/

17. exp ligation/ or exp diathermy/
18. exp carotid artery, external/

19. exp maxillary artery/

20. exp surgical instruments/

21. exp laser therapy/

22. exp postoperative complications/

23. or $/ 11-22$

24. 10 and 23

25. (case adj report*).ti,ab.

26. exp case reports/

27. case reports.pt.

28. or $/ 25-27$

29. $24 \operatorname{not} 28$

\section{Embase strategy}

1. exp epistaxis/

2. (epistax* or nosebleed* ${ }^{*}$ or rhinorrhag* or rhinorrhaeg*).ti,ab.

3. $\exp$ nose/

4. (nose* $^{*}$ or nasal* ${ }^{*}$ or nostril ${ }^{*}$ or nasopharyn* or olfactory).ti,ab.

5. 3 or 4

6. exp bleeding/

7. (hemorrhag* or haemorrhag* or bleed* or bloodloss* or (blood adj3 loss)).ti,ab.

8. 6 or 7

9. 5 and 8

10. 1 or 2 or 9

11. (surger* or surgic* or operat* or ligat* or transoral $^{*}$ or transnasal* or Caldwell-luc* or diatherm* or post-operat* or pre-operat* or postsurg* or pre-surg* or sphenopalatine or ethmoid or (internal adj maxillary adj arter*) or (external adj carotid adj arter*) or clip* or staple* or laser*).ti,ab.

12. 10 and 11

13. (case adj report*).ti,ab.

14. case report/

15. 13 or 14

16. $12 \operatorname{not} 15$

\section{Cochrane Database of Systematic Reviews}

1. MeSH descriptor: [Epistaxis] explode all trees

2. epistax* or nosebleed* or rhinorrhag* or rhinorrhaeg*:ti,ab,kw

3. MeSH descriptor: [Nose] explode all trees

4. nose* or nasal* or oronasal or nostril* ${ }^{*}$ or nasopharynx* or olfactory:ti,ab,kw

5. \#3 or \#4

6. MeSH descriptor: [Hemorrhage] explode all trees

7. hemorrhag* or haemorrhag* or bleed* or bloodloss* or (blood near loss):ti,ab,kw

8. \#6 or \#7

9. \#5 and \#8

10. $\# 1$ or $\# 2$ or $\# 9$

11. MeSH descriptor: [General Surgery] explode all trees 
12. MeSH descriptor: [Surgical Procedures, Operative] explode all trees

13. MeSH descriptor: [Postoperative Care] explode all trees

14. MeSH descriptor: [Postoperative Period] explode all trees

15. MeSH descriptor: [Preoperative Period] explode all trees

16. MeSH descriptor: [Preoperative Care] explode all trees

17. MeSH descriptor: [Ligation] explode all trees

18. MeSH descriptor: [Diathermy] explode all trees

19. MeSH descriptor: [Carotid Arteries] explode all trees

20. MeSH descriptor: [Maxillary Artery] explode all trees

21. MeSH descriptor: [Surgical Instruments] explode all trees

22. MeSH descriptor: [Laser Therapy] explode all trees

\begin{tabular}{l} 
23. $\mathrm{MeSH}$ descriptor: [Postoperative \\
\hline
\end{tabular} Complications] explode all trees

24. (surger* or surgic* or operat* or ligat* or transoral $^{*}$ or transnasal ${ }^{*}$ or Caldwell-luc* or diatherm* or post-operat* or pre-operat* or postsurg* or pre-surg* or sphenopalatine or ethmoid or ("internal maxillary arter*") or ("external carotid arter*") or clip* or staple* or laser*):ti,ab,kw (Word variations have been searched)

25 . \#11 or \#12 or \#13 or \#14 or \#15 or \#16 or \#17 or $\# 18$ or \#19 or \#20 or \#21 or \#22 or \#23 or \#24

26. \#10 and \#25

\section{CINAHL via EbscoHost}

1. (MH "Epistaxis")

2. TI (epistax* or nosebleed* or rhinorrhag* or rhinorrhaeg*) OR AB (epistax* or nosebleed* or rhinorrhag* or rhinorrhaeg*)

3. (MH "Nose+")

4. TI (nose* or nasal ${ }^{*}$ or oronasal or nostril ${ }^{*}$ or nasopharynx* or olfactory) OR AB (nose* or nasal* $^{*}$ or oronasal or nostril* or nasopharynx* or olfactory)

5. S3 OR S4

6. (MH "Hemorrhage+")

7. TI (hemorrhag* or haemorrhag* or bleed* or bloodloss* or (blood N3 loss)) OR AB (hemorrhag* or haemorrhag* or bleed* or bloodloss* or (blood N3 loss))

8. S6 OR S7

9. S5 AND S8

10. S1 OR S2 OR S9

11. (MH "Postoperative Complications+")

12. (MH "Postoperative Period")

13. (MH "Postoperative Care+")

14. (MH "Preoperative Period+")

15. (MH "Preoperative Care+")
16. (MH "Ligation")

17. (MH "Diathermy+")

18. (MH "Carotid Arteries")

19. (MH "Maxillary Artery")

20. (MH "Surgical Instruments")

21. (MH "Laser Therapy+")

22. (MH "Surgery, Operative+")

23. TI ((surger* or surgic* or operat* or ligat* or transoral $^{*}$ or transnasal* or Caldwell-luc* or diatherm* or post-operat* or pre-operat* or postsurg* $^{*}$ or pre-surg* or sphenopalatine or ethmoid or ("internal maxillary arter"”) or ("external carotid arter*") or clip* or staple* or laser*)) OR AB ((surger* or surgic* or operat* or ligat* or transoral* or transnasal ${ }^{*}$ or Caldwell-luc* or diatherm* or post-operat* or pre-operat* or post-surg* or pre-surg* or sphenopalatine or ethmoid or ("internal maxillary arter*") or ("external carotid arter*") or clip* or staple* or laser*))

24. S11 or S12 OR S13 OR S14 OR S15 OR S16 OR S17 OR S18 OR S19 OR S20 OR S21 OR S22 OR S23

25. S10 AND S24

\section{Web of Science}

1. $\mathrm{Ts}=\left(\right.$ epistax $^{*}$ or nosebleed* or rhinorrhag* or rhinorrhaeg* or ((nose* or nasal* or oronasal or nostril ${ }^{*}$ or nasopharynx* or olfactory) and (hemorrhag* or haemorrhag* or bleed* or bloodloss* or (blood N/3 loss))))

2. $\mathrm{TS}=\left(\left(\left(\right.\right.\right.$ surger*$^{*}$ or surgic* or operat* or ligat* $^{*}$ or transoral $^{*}$ or transnasal* or Caldwell-luc* or diatherm* or post-operat* or pre-operat* or postsurg* or pre-surg* or sphenopalatine or ethmoid or ("internal maxillary arter") or ("external carotid arter*") or clip* or staple* or laser*)))

3. 1 AND 2

\section{Epistaxis and radiology}

All databases searched 12th February 2016, no limits applied.

\section{Medline via Ovid}

1. exp Epistaxis/

2. (epistax* or nosebleed* or rhinorrhag* or rhinorrhaeg*).ti,ab.

3. $\exp$ Nose/

4. (nose* $^{*}$ or nasal ${ }^{*}$ or oronasal or nostril ${ }^{*}$ or nasopharynx* or olfactory).ti,ab.

5. 3 or 4

6. exp Hemorrhage/

7. (hemorrhag* or haemorrhag* or bleed* or bloodloss* or (blood adj3 loss)).ti,ab.

8. 6 or 7

9. 5 and 8

10. 1 or 2 or 9 
11. (radiolog* or angiograph* or arteriograph* or embolis* or emboliz* or endovascular* or coil* or fluoroscop* or atherectom*).ti,ab.

12. exp radiology/ or exp angiography/

13. exp embolization, therapeutic/

14. exp fluoroscopy/

15. exp endovascular procedures/

16. or $/ 11-15$

17. 10 and 16

\section{Embase strategy}

1. exp epistaxis/

2. (epistax* or nosebleed* or rhinorrhag* or rhinorrhaeg*).ti,ab.

3. exp nose/

4. (nose* or nasal* or nostril* ${ }^{*}$ or nasopharyn* or olfactory).ti,ab.

5. 3 or 4

6. $\exp$ bleeding/

7. (hemorrhag* or haemorrhag* or bleed* or bloodloss* or (blood adj3 loss)).ti,ab.

8. 6 or 7

9. 5 and 8

10. 1 or 2 or 9

11. (radiolog* or angiograph* or arteriograph* or embolis* or emboliz* or endovascular* or coil* or fluoroscop* or atherectom*).ti,ab.

12. 10 and 11

\section{Cochrane Database of Systematic Reviews}

1. MeSH descriptor: [Epistaxis] explode all trees

2. epistax* or nosebleed* or rhinorrhag* or rhinorrhaeg*:ti,ab,kw

3. MeSH descriptor: [Nose] explode all trees

4. nose* or nasal* or oronasal or nostril ${ }^{*}$ or nasopharynx* or olfactory:ti,ab,kw

5. \#3 or \#4

6. MeSH descriptor: [Hemorrhage] explode all trees

7. hemorrhag* or haemorrhag* or bleed* or bloodloss* or (blood near loss):ti,ab,kw

8. \#6 or \#7

9. $\# 5$ and $\# 8$

10. \#1 or \#2 or \#9

11. radiolog* or angiograph* or arteriograph* or embolis* or emboliz* or endovascular* or coil ${ }^{*}$ or fluoroscop* or atherectom*:ti,ab,kw

12. MeSH descriptor: [Radiology] explode all trees

13. MeSH descriptor: [Angiography] explode all trees

14. MeSH descriptor: [Embolization, Therapeutic] explode all trees\#
15. MeSH descriptor: [Fluoroscopy] explode all trees

16. MeSH descriptor: [Endovascular Procedures] explode all trees

17. $\# 11$ or $\# 12$ or $\# 13$ or $\# 14$ or $\# 15$ or $\# 16$

18. \#10 and \#17

\section{CINAHL via EbscoHost}

1. (MH "Epistaxis")

2. TI (epistax* or nosebleed* or rhinorrhag* or rhinorrhaeg*) OR AB (epistax* or nosebleed* or rhinorrhag* or rhinorrhaeg*)

3. (MH "Nose+")

4. TI (nose* or nasal* or oronasal or nostril* or nasopharynx* or olfactory) OR AB (nose* or nasal* $^{*}$ or oronasal or nostril* or nasopharynx* or olfactory)

5. S3 OR S4

6. (MH "Hemorrhage+")

7. TI (hemorrhag* or haemorrhag* or bleed* or bloodloss* or (blood N3 loss)) OR AB (hemorrhag* or haemorrhag* or bleed* or bloodloss* or (blood N3 loss))

8. S6 OR S7

9. S5 AND S8

10. S1 OR S2 OR S9

11. (MH "Radiology Service")

12. (MH "Radiological Nursing")

13. (MH "Angiography+")

14. (MH "Embolization, Therapeutic+")

15. (MH "Fluoroscopy")

16. TI (radiolog* or angiograph* or arteriograph* or embolis* or emboliz* or endovascular* or coil* or fluoroscop* or atherectom*) OR AB (radiolog* or angiograph* or arteriograph* or embolis* or emboliz* or endovascular* or coil ${ }^{*}$ or fluoroscop* or atherectom*)

17. S11 OR S12 OR S13 OR S14 OR S15 OR S16

18. S10 AND S17

Web of Science

1. $\mathrm{TS}=\left(\right.$ epistax $^{*}$ or nosebleed ${ }^{*}$ or rhinorrhag* or rhinorrhaeg* or ((nose* or nasal* or oronasal or nostril* or nasopharynx* or olfactory) and (hemorrhag* or haemorrhag* or bleed* or bloodloss* or (blood N/3 loss))))

2. $\mathrm{TS}=\left(\right.$ radiolog* $^{*}$ or angiograph ${ }^{*}$ or arteriograph* or embolis* or emboliz* or endovascular* or coil* $^{*}$ or fluoroscop* or atherectom*)

3. 1 and 2 